\title{
Fluorescence Recovery of GFP Emission in Samples Embedded for TEM Upon Exposure to an Alkaline Solution
}

\author{
Douglas R. Keene ${ }^{1}$, Sunjong Kwon ${ }^{2}$, Sara F. Gonzales ${ }^{3}$, and Claudia S. López ${ }^{4}$ \\ 1,3 Shriners Hospital for Children, Micro-Imaging Center, Portland, Oregon \\ 1,2,4, Department of Biomedical Engineering, OHSU Center for Spatial Systems Biomedicine, Oregon \\ Health Sciences University, Portland, Oregon
}

Fluorescence Microscopy (FM) localization of GFP derivatives provides information - sometimes dynamic - about the distribution of proteins in cells and tissues while electron microscopy provides information about the ultrastructural components of cells. Correlative light and electron microscopy (CLEM) integrates these two complementary imaging techniques generating a more complete view of the micro- and nanostructure of cells and tissues.

Receptor tyrosine kinase (RTK)-transduced signaling pathways are highly activated in breast cancer and have been developed as therapeutic targets. The EGFR-family of RTKs includes Her2 receptors which function to regulate cell behavior in response to the extracellular environment, and Her3, essential for Her2-driven tumorigenesis $(1,2)$. Imaging studies have shown that Her2 is preferentially localized to specialized membrane protrusions of actin-rich filopodia-like structures (FLPs) (3) which may serve as sites for ligand binding and consequent initiation of signaling pathways.

Here we present a correlative FM-TEM workflow involving two fluorescently tagged membrane proteins (Her2 and Her3) associated with drug resistance in human breast cancer. SKBR3 breast cancer cells were grown in 35mm culture dish and were transiently co-transfected with 500ng of DNA of each Her2-eGFP and Her3-mRFP expression plasmids and seeded onto Thermanox coverslips (13mm). Cells were fixed in $1 \%$ glutaraldehyde for $25 \mathrm{~min}$ on ice, rinsed in DMEM then in Tris-HCl with $0.05 \mathrm{M}$ glycine overnight on ice, then dehydrated in 30,50, 70, 90\% ethanol series on ice for 10 min each, then infiltrated in LR Gold, $\mathrm{pH}$ 8.7. The final change of LR Gold included $0.2 \%$ water and $0.5 \%$ benzoin methyl ether. UV polymerization was in a nitrogen atmosphere at $-20 \mathrm{C}$ a utilizing a Leica AFS (4).

0.5 to 1.0 micron sections were collected onto glass coverslips; the next serial ultrathin section was collected onto a formvar coated $1 \times 2 \mathrm{~mm}$ slot grid. Sequential imaging of GFP and RFP was at excitation wavelength $488 \mathrm{~nm}$ and $543 \mathrm{~nm}$ using a Leica TCS SP4 laser confocal system fitted with an inverted microscope. Initial imaging of dry $0.5-1.0$ micron thick sections mounted on glass cover slips demonstrated a dull green background with 488nm excitation wavelength. Sodium carbonate, $\mathrm{pH} 11$ placed dropwise onto the section during imaging resulted in an immediate decrease in background with a simultaneous increase in Her2-eGFP localization to the cell membrane of occasional cells within the culture (Fig 1), consistent with expected transfection rate. 543nm excitation wavelength resulted in both membrane and cytoplasmic localization of Her3-mRFP on dry sections with no significant intensity change after sodium carbonate (Fig 2). Water substituted for sodium carbonate had no effect on fluorescence emission. Our results indicate that 1) GFP and RFP endured our chemical fixation and embedding procedures; 2) For fluorescence imaging, the hydration state of embedded GFP proteins is not as crucial as is $\mathrm{pH}$; 3) Detection of GFP during "in block face" imaging may be improved by the dropwise addition of alkaline solutions, such as sodium carbonate $\mathrm{pH} 11.0$; 4) RFP signal is not improved after addition of alkaline solutions. 


\section{References:}

[1] Holbro T et al, CF 3rd, Hynes NE (2003) Proc Natl Acad Sci USA 100(15):8933-8938 15, 2003. [2] Lee-Hoeflich ST et al, Cancer Res 68(14):5878-5887, 2008.

[3] Anette M. Hommelgaard, Mads Lerdrup and Bo van Deurs. Mol Biol Cell. Apr; 15(4): 1557-1567, 2004.

[4] Keene et al., in Correlative Light and Electron Microscopy II, ed. Thomas Muller-Reichert \& Paul Verkade, Elsevier, New York, pp 391-416.
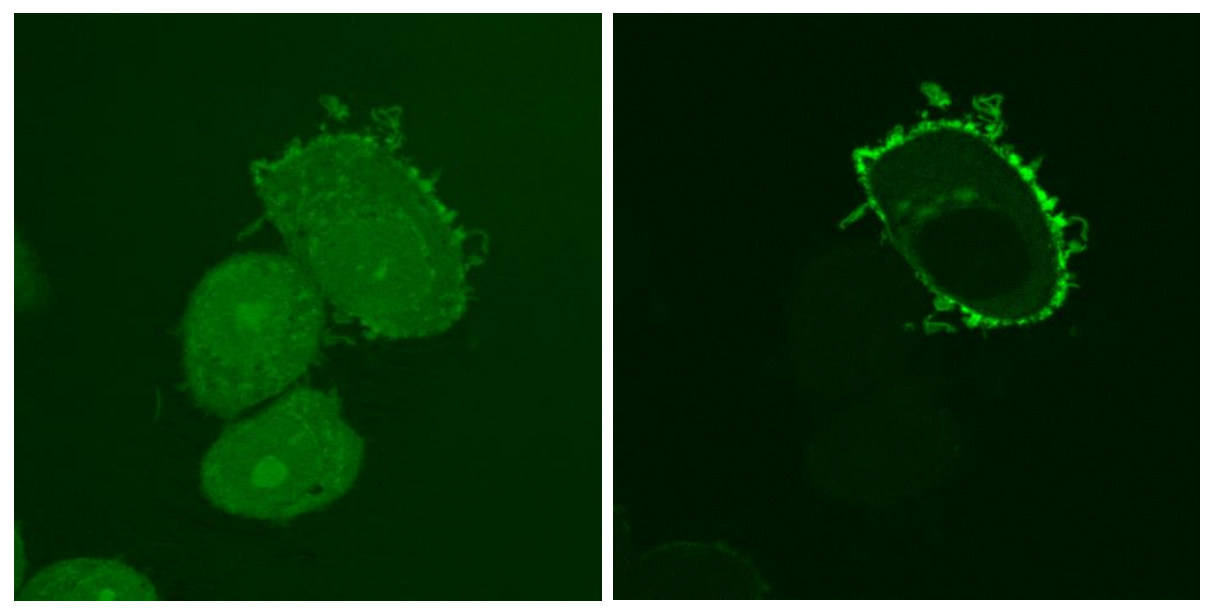

Figure 1. Prior to adding a drop of sodium carbonate to the section, all cells within the culture had a dull green appearance. Following alkaline treatment background flourescence diminished with a simultaneous increase in Her2e-GFP plasma membrane staining.
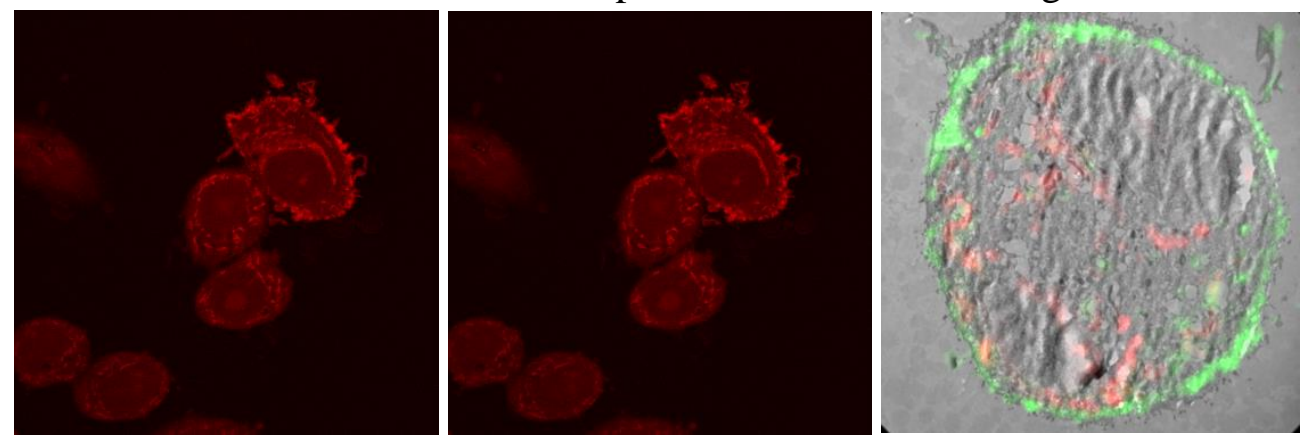

Figure 2. The Her3-mRFP staining pattern did not change in intensity in response to sodium carbonate. FM images may be overlaid onto TEM images collected from a serial ultrathin section image, allowing ultrastructural detail of stained regions. 